\title{
Invertible and Nilpotent Elements in the Group Algebra of a Unique Product Group
}

\author{
Erhard Neher
}

Received: 20 October 2008 / Accepted: 4 November 2008 / Published online: 18 November 2008

(C) Springer Science+Business Media B.V. 2008

\begin{abstract}
We describe the nilpotent and invertible elements in group algebras $k[G]$ for $k$ a commutative associative unital ring and $G$ a unique product group, for example an ordered group.
\end{abstract}

Keywords Units · Nilpotent elements · Invertible elements

Mathematics Subject Classification (2000) Primary 16S34 · Secondary 06F15 · 16 U60 • 20F60

\section{Introduction}

A fundamental problem in the theory of group algebras is to determine their units $=$ invertible elements. The reader can find a short introduction to this question in $[3, \S 6]$ and a much more substantial one in [6, Chap. 13] and [7, Chaps. II and VI]. Since $1-a$ is invertible for any nilpotent element $a$, a closely related problem is that of describing all nilpotent elements.

In this short note we give a description of the nilpotent and invertible elements in group algebras $k[G]$ where $k$ is an arbitrary commutative associative unital ring and $G$ is a unique product group, e.g. an ordered group (Corollary 6). Our result is well-known in case $k$ is an integral domain: If $G$ is a unique product group, 0 is the only nilpotent element and all units are trivial. So the main point here is the generality of $k$.

Our approach uses a little bit of algebraic geometry and might possibly also be of interest to solve other problems related to group algebras. It is inspired by a recent result of Ottmar Loos in [4], where he determines the invertible elements in a Laurent polynomial ring $k\left[t^{ \pm 1}\right]$. In Theorem 3 we describe the nilpotent and invertible elements in $k[G]$ under

The author was partially supported by a Natural Sciences and Engineering Research Council of Canada Discovery Grant.

E. Neher $(\bowtie)$

Department of Mathematics and Statistics, University of Ottawa, Ottawa, Ontario K1N 6N5, Canada

e-mail: neher@uottawa.ca 
the assumption that for all $k$-algebras $K$ which are fields the group algebra $K[G]$ is a domain or, respectively, has only the trivial units. The case of group algebras $k[G]$ for $G$ a unique product group is then an immediate corollary.

A different characterization of the units in $k[G]$ for $G$ a right-ordered and thus unique product group is proven in [5].

\section{Notation}

Throughout we use the following notation: $k$ is a commutative associative unital ring, $\operatorname{Spec}(k)$ is the prime spectrum of $k$ equipped with the Zariski topology, $\kappa(\mathfrak{p})$ is the quotient field of $k / \mathfrak{p}$ for $\mathfrak{p} \in \operatorname{Spec}(k), x(\mathfrak{p})$ is the canonical image of $x \in k$ in $\kappa(\mathfrak{p})$ and $k$-alg is the category of associative commutative and unital $k$-algebras. The invertible elements of an associative unital $k$-algebra $A$ are denoted $A^{\times}$.

Let $G$ be a group, written multiplicatively and let $A=k[G]$ be the group algebra of $G$ over $k$. Thus $A$ is a free $k$-module with $k$-basis $\left(u_{g}: g \in G\right)$ in bijection with $G$ by $g \mapsto u_{g}$ and the multiplication of the $k$-algebra $A$ is determined by the rule $u_{g} u_{h}=u_{g h}$ for $g, h \in G$. It is immediate that any element $x u_{g}, x \in k^{\times}$is invertible. These are the so-called trivial units of $A$.

We endow $G$ with the discrete topology. Recall the definition of the constant group scheme $\mathbf{G}$ associated to $G$ [2, II, §1, no. 2.12]: For $R \in k$-alg, $\mathbf{G}(R)$ is the set of continuous (= locally constant) maps $\mathfrak{d}: \operatorname{Spec}(R) \rightarrow G$ with the group structure inherited from $G$. In particular, this applies to $R=k$.

Since $\operatorname{Spec}(k)$ is quasi-compact [1, II, §4.3, Proposition 12], it follows from [1, II, §4.3, Proposition 15] that there exists a bijection $\mathcal{I}: \mathbf{G}(k) \rightarrow \mathcal{E}$ from $\mathbf{G}(k)$ to the set $\mathcal{E}$ of all families $\varepsilon=\left(\varepsilon_{g}\right)_{g \in G}$ of orthogonal idempotents in $k$ with $\varepsilon_{g} \neq 0$ for only finitely many $g \in G$ and $\sum_{g \in G} \varepsilon_{g}=1_{k}$. The bijection $\mathfrak{d} \mapsto \mathcal{I}(\mathfrak{d})=\left(\varepsilon_{g}\right)_{g \in G}$ is given by the relations

$$
\mathfrak{d}(\mathfrak{p})=g \quad \Longleftrightarrow \varepsilon_{g} \notin \mathfrak{p} \quad \Longleftrightarrow \mathfrak{p} \in(\operatorname{Spec}(k))_{\varepsilon_{g}} \quad \Longleftrightarrow \varepsilon_{g}(\mathfrak{p})=1_{\kappa(\mathfrak{p})}
$$

where, for $x \in k,(\operatorname{Spec}(k))_{x}$ denotes the basic open subset of all $\mathfrak{p} \in \operatorname{Spec}(k)$ with $x \notin \mathfrak{p}$. We will usually view $\mathcal{I}$ as an identification. The product of $\varepsilon=\left(\varepsilon_{g}\right)$ and $\varepsilon^{\prime}=\left(\varepsilon_{g}^{\prime}\right)$ in the group $\mathbf{G}(k)$ is then given by the formula

$$
\left(\varepsilon \cdot \varepsilon^{\prime}\right)_{x}=\sum_{g h=x} \varepsilon_{g} \varepsilon_{h}^{\prime} \quad(x \in G) .
$$

Indeed, a locally constant function $\mathfrak{d}: \operatorname{Spec}(k) \rightarrow k$ gives rise to a partition of $\operatorname{Spec}(k)$ by basic open sets $\operatorname{Spec}\left(k \varepsilon_{g}\right)=\operatorname{Spec}(k)_{\varepsilon_{g}}$, where $\varepsilon=\left(\varepsilon_{g}\right)$ is the complete orthogonal system corresponding to $\mathfrak{d}$ and where $\operatorname{Spec}(k)_{x}$ is canonically identified with a subset of $\operatorname{Spec}(k)$. Given two locally constant functions $\mathfrak{d}$ and $\mathfrak{d}^{\prime}$ with corresponding orthogonal systems $\varepsilon=$ $\mathcal{I}(\mathfrak{d})$ and $\varepsilon^{\prime}=\mathcal{I}\left(\mathfrak{d}^{\prime}\right)$ we get a partition of $\operatorname{Spec}(k)$ by open sets

$$
(\operatorname{Spec}(k))_{\varepsilon_{g}} \cap(\operatorname{Spec}(k))_{\varepsilon_{h}^{\prime}}=(\operatorname{Spec}(k))_{\varepsilon_{g} \varepsilon_{h}^{\prime}}=\operatorname{Spec}\left(k \varepsilon_{g} \varepsilon_{h}^{\prime}\right)
$$

on which the function $\mathfrak{d} \mathfrak{d}^{\prime}$ has the value $g h$. Hence $\mathfrak{d} \mathfrak{d}^{\prime}$ has value $x \in G$ precisely on

$$
\bigcup_{g h=x} \operatorname{Spec}\left(k \varepsilon_{g} \varepsilon_{h}^{\prime}\right)=\operatorname{Spec}\left(k\left(\sum_{g h=x} \varepsilon_{g} \varepsilon_{h}^{\prime}\right)\right) .
$$


In terms of the $\varepsilon^{\prime}$ s, the unit element of $\mathbf{G}(k)$ is the family $\varepsilon^{(0)}=\left(\varepsilon_{g}^{(0)}\right)$ with

$$
\varepsilon_{g}^{(0)}= \begin{cases}1_{k}, & g=1_{G} \\ 0, & g \neq 1_{G}\end{cases}
$$

and the inverse of $\varepsilon=\left(\varepsilon_{g}\right)_{g \in G}$ is $\varepsilon^{-1}=\left(\varepsilon_{g}^{-1}\right)_{g \in G}$ with $\varepsilon_{g}^{-1}=\varepsilon_{g^{-1}}$.

Let now $A=k[G]$ be the group algebra of $G$. We then have a group monomorphism

$$
\mathbf{G}(k) \rightarrow k[G]^{\times}, \quad \mathfrak{d} \mapsto u_{\mathfrak{d}}:=\sum_{g \in G} \varepsilon_{g} u_{g}, \quad \text { for } \varepsilon=\mathcal{I}(\mathfrak{d}) .
$$

Indeed, it follows from (2) that $u_{\mathfrak{d}} u_{\mathfrak{d}^{\prime}}=u_{\mathfrak{d} \mathfrak{d}^{\prime}}$ for all $\mathfrak{d}, \mathfrak{d}^{\prime} \in \mathbf{G}(k)$.

We recall that a nil ideal of an associative algebra $A$ is an ideal consisting of nilpotent elements. By definition $[3,10.26]$, the upper nil radical of an associative algebra $A$ is the sum $\mathrm{Nil}^{*}(A)$ of all nil ideals of $A$, equivalently, $\mathrm{Nil}^{*}(A)$ is the biggest nil ideal of $A$. If $A$ is also commutative, $\operatorname{Nil}^{*}(A)=\{a \in A: a$ nilpotent $\}=\operatorname{Nil}(A)$, the nil radical of $A$.

Theorem 3 (a) Assume $K[G]$ is a domain for every field $K \in k$-alg. Then the upper nil radical of $k[G]$ is

$$
\operatorname{Nil}^{*}(k[G])=\left\{\sum_{g \in G} n_{g} u_{g}: n_{g} \in \operatorname{Nil}(k) \text { for every } g \in G\right\} \cong(\operatorname{Nil}(k))[G] .
$$

It coincides with the set of nilpotent elements of $k[G]$.

(b) Suppose that $K[G]$ has only trivial units whenever $K \in k$-alg is a field. Then an element $a \in k[G]$ is invertible if and only if there exists $\mathfrak{d} \in \mathbf{G}(k)$, a unit $v \in k^{\times}$and an element $n \in \operatorname{Nil}^{*}(k[G])$ such that

$$
a=v u_{\mathfrak{d}}+n \quad\left(v \in k^{\times}, \mathfrak{d} \in \mathbf{G}(k), n \in \operatorname{Nil}^{*}(k[G])\right) .
$$

The element $\mathfrak{d}$ is uniquely determined by a, called the degree of $a$ and the map

$$
\operatorname{deg}: k[G]^{\times} \rightarrow \mathbf{G}(k), \quad \operatorname{deg}\left(v u_{\mathfrak{d}}+n\right)=\mathfrak{d}
$$

is a group homomorphism.

Proof (a) We abbreviate $A=k[G]$. It is easily seen that $\mathfrak{N}:=\left\{\sum_{g \in G} n_{g} u_{g}: n_{g} \in\right.$ $\operatorname{Nil}(k)$ for every $g \in G\}$ is an ideal of $A$ consisting of nilpotent elements. Indeed, as an element of $A$, an $n \in \mathfrak{N}$ has only finitely many non-zero components, say $n_{1} u_{g_{1}}, \ldots, n_{p} u_{g_{p}}$. Hence there exists $q \in \mathbb{N}$ such that $n_{i}^{q}=0$ for all $1 \leq i \leq p$. Then $n^{p q}$ is a sum of terms $n_{1}^{r_{1}} \cdots n_{p}^{r_{p}} u_{g}$ where $g$ is an appropriate product of $p q$ factors taken from the $g_{1}, \ldots, g_{r}$ and where at least one $r_{i} \geq q$. Thus $n^{p q}=0$. Hence $\mathfrak{N} \subset$ Nil$^{*}(A)$ (observe that this holds in general).

To finish the proof of (a), it is now sufficient to show $n \in \mathfrak{N}$ for every nilpotent element $n$ of $A$. We write $n=\sum_{g \in G} n_{g} u_{g}$ with $n_{g} \in k$ and let $\mathfrak{p} \in \operatorname{Spec}(k)$. The element $n(\mathfrak{p}) \in$ $A \otimes_{k} \kappa(\mathfrak{p}) \cong(\kappa(\mathfrak{p}))[G]$ is then nilpotent too. But since by assumption $\kappa(\mathfrak{p})[G]$ is a domain, it follows that $n(\mathfrak{p})=0$, i.e., $n_{g}(\mathfrak{p})=0$ for all $\mathfrak{p} \in \operatorname{Spec}(k)$ and all $g \in G$. Thus, every $n_{g}$ is nilpotent and $n \in \mathfrak{N}$.

(b) We will first show that any element of the form (4) is invertible. This is clear for $v u_{\varepsilon}$, so that it suffices to prove invertibility of $\left(v u_{\varepsilon}\right)^{-1} a=1+v^{-1} u_{\delta} n$ for $\delta=\varepsilon^{-1}$. But this is clear since $v^{-1} u_{\delta} n \in \mathfrak{N}$ is nilpotent. 
Conversely, suppose that $a \in k[G]$ is invertible. If $k$ is a field, $a$ has the form $a=v u_{g}$ for some $v \in k^{\times}$by assumption, which is a special case of (4).

Let now $k$ be arbitrary. We write $a=\sum_{g \in G} a_{g} u_{g}$ with $a_{g} \in k$. Let $\mathfrak{p} \in \operatorname{Spec}(k)$. Then there exits a unique $g \in G$ such that $a(\mathfrak{p})=a_{g}(\mathfrak{p}) u_{g} \neq 0$. This gives rise to a map $\mathfrak{d}: \operatorname{Spec}(k) \rightarrow G$ which, we claim, is locally constant. Indeed, if $\mathfrak{d}\left(\mathfrak{p}_{0}\right)=g$ then $a_{g}(\mathfrak{p}) \neq 0$ and hence $a_{g}(\mathfrak{p}) \neq 0$ for all $\mathfrak{p}$ in the basic open neighborhood $U=(\operatorname{Spec}(k))_{x}, x=a_{g}$. Since then $a_{h}(\mathfrak{p})=0$ for all $h \neq g$ and $\mathfrak{p} \in U$, we see that $\mathfrak{d}$ is constant equal to $g$ on $U$. Thus $\mathfrak{d} \in \mathbf{G}(k)$.

Let $\varepsilon=\left(\varepsilon_{g}\right)_{g \in G}$ be the family corresponding to $\mathfrak{d}$. Then $\left(a_{g}\left(1-\varepsilon_{g}\right)\right)(\mathfrak{p})=0$ for all $\mathfrak{p} \in \operatorname{Spec}(k)$. Indeed, if $\mathfrak{d}(\mathfrak{p})=g$ then $\left(1-\varepsilon_{g}\right)(\mathfrak{p})=1_{\kappa(\mathfrak{p})}-1_{\kappa(\mathfrak{p})}=0$ by (1), while if $\mathfrak{d}(\mathfrak{p}) \neq g$ then $a_{g}(\mathfrak{p})=0$ by definition of $\mathfrak{d}$. Hence $n_{g}=a_{g}\left(1-\varepsilon_{g}\right) \in k$ is nilpotent. Also $v=\sum_{g \in G} a_{g} \varepsilon_{g} \in k^{\times}$since, for any $\mathfrak{p} \in \operatorname{Spec}(k), v(\mathfrak{p})=\sum_{g \in G} a_{g}(\mathfrak{p}) \varepsilon_{g}(\mathfrak{p})=a_{\mathfrak{d}(\mathfrak{p})}(\mathfrak{p}) \neq 0$. Thus,

$$
a=\sum_{g \in G} a_{g} \varepsilon_{g} u_{g}+\sum_{g \in G} a_{g}\left(1-\varepsilon_{g}\right) u_{g}=\left(\sum_{g \in G} a_{g}\right)\left(\sum_{h \in G} \varepsilon_{h} u_{h}\right)+n
$$

as required in (4).

Uniqueness of $\mathfrak{d}$, i.e. of $\varepsilon$, is clear from the construction above. For the proof of the last claim, let $a^{\prime}=v^{\prime} u_{\varepsilon^{\prime}}+n^{\prime}$ be another invertible element of $A$. Then $a a^{\prime}=v v^{\prime} u_{\varepsilon \varepsilon^{\prime}}+b$ where $b=v u_{\varepsilon} n^{\prime}+v^{\prime} u_{\varepsilon^{\prime}} n^{\prime}+n n^{\prime} \in \mathfrak{N}$. Thus $a a^{\prime}$ has degree $\varepsilon \varepsilon^{\prime}=\mathcal{I}\left(\mathfrak{d} \mathfrak{d}^{\prime}\right)$ proving that deg is a homomorphism.

Example 4 Suppose $k$ is reduced (= semiprime) and that $K[G]$ has only trivial units for any field $K \in k$-alg. Then Theorem 3 says that for any unit $a \in k[G]$ there exists a decomposition of $k$ into a finite direct sum of ideals $I$ such that $a$ decomposes into trivial units in each $I[G]$.

\section{Unique Product Groups}

It is well-known that the assumptions in (a) and (b) of Theorem 3 are fulfilled for ordered groups, see for example [3, Theorem 6.29]. However, they are also fulfilled for the much more general class of so-called unique product groups.

Recall [6] that a group $G$ is called a unique product group, abbreviated u.p. group, if, given any two finite non-empty subsets $A, B$ of $G$, there is an element of $A B$ that can be uniquely written in the form $a b$ with $a \in A$ and $b \in B$. It follows immediately [6,13, Lemma 1.9(i)] that if $R$ is an integral domain and $G$ is a u.p. group then $R[G]$ is a domain. Furthermore, it is also known [6, Appendix, Theorem 15] that a u.p. group $G$ is the same as a two unique products group: if $A, B \subset G$ are finite non-empty subsets, not both singletons, there are at least two elements in $A B$ which are uniquely represented. It then follows $[6,13$, Lemma 1.9(ii)] that any unit in $R[G], R$ an integral domain, is trivial. To summarize:

Corollary 6 If $G$ is a u.p. group, e.g. an ordered group, the assumptions in (a) and (b) are fulfilled. Hence (3) and (4) describe the nilpotent and invertible elements of the group algebra $k[G]$.

Acknowledgements The author thanks Ottmar Loos, Donald Passman and Sudarshan Sehgal for very useful comments on earlier versions of this paper. In particular, it was Donald Passman who pointed out that the main result of this note, originally stated only for ordered groups, actually holds for u.p. groups. 


\section{References}

1. Bourbaki, N.: Éléments de mathématique. Fascicule XXVII. Algèbre commutative. Chapitre 1: Modules plats. Chapitre 2: Localisation. Actualités Scientifiques et Industrielles, vol. 1290. Herman, Paris (1961)

2. Demazure, M., Gabriel, P.: Groupes algébriques. Tome I: Géométrie algébrique, généralités, groupes commutatifs. Masson, Paris (1970). Avec un appendice it Corps de classes local par Michiel Hazewinkel

3. Lam, T.Y.: A First Course in Noncommutative Rings. Graduate Texts in Mathematics, vol. 131. Springer, New York (1991)

4. Loos, O.: Remarks on Holger P. Petersson's paper "Idempotent 2-by-2 matrices". Mathematikberichte, Universität Hagen 78 (2007), see also Jordan Theory Preprint Archive, paper 243

5. Parmenter, M.M.: Units and isomorphism in group rings. Quaest. Math. 8(1), 9-14 (1985)

6. Passman, D.S.: The Algebraic Structure of Group Rings. Robert E. Krieger Publishing Co. Inc., Melbourne (1985), Reprint of the 1977 original

7. Sehgal, S.K.: Topics in Group Rings. Monographs and Textbooks in Pure and Applied Math., vol. 50. Dekker, New York (1978) 